\title{
A contemporary assessment of devices for Resuscitative Endovascular Balloon Occlusion of the Aorta (REBOA): resource-specific options per level of care
}

\author{
Suzanne M. Vrancken ${ }^{1}$ (D) - Boudewijn L. S. Borger van der Burg ${ }^{1}$ · Paul J. E. M. Vrancken ${ }^{1}$. Gert-Aldert H. Kock ${ }^{2}$. \\ Todd E. Rasmussen ${ }^{3} \cdot$ Rigo Hoencamp ${ }^{1,4,5,6}$
}

Received: 31 January 2020 / Accepted: 24 April 2020

(c) Springer-Verlag GmbH Germany, part of Springer Nature 2020

\begin{abstract}
Purpose Use of Resuscitative Endovascular Balloon Occlusion of the Aorta (REBOA) as adjunct for temporary hemorrhage control in patients with exsanguinating torso hemorrhage is increasing. Characteristics of aortic occlusion balloons (AOB) are diverse and evolving as efforts are made to improve the technology. It is important to select a device that fits the requirements of the medical situation to minimize the risk of failure and complications. The aim of this study is to appraise guidance in the choice of an AOB in a specific situation.

Methods We assessed 29 AOB for differences and outline possible advantages and disadvantages of each. Bending stiffness was measured with a three-point bending device.

Results Diameter of the AOB ranged from $6\left(\right.$ ER-REBOA $\left.^{\mathrm{TM}}\right)$ to $10\left(\mathrm{Coda}^{\circledR}-46\right)$ French. However, some need large-bore access sheaths up to 22 French (Fogarty ${ }^{\circledR}-45$ and LeMaitre ${ }^{\circledR}-45$ ) or even insertion via cut-down (Equalizer ${ }^{\text {TM}}-40$ ). Bending stiffness varied from $0.08 \mathrm{~N} / \mathrm{mm}\left( \pm 0.008 \mathrm{SD}\right.$; Coda $\left.{ }^{\circledR}-32\right)$ to $0.72 \mathrm{~N} / \mathrm{mm}\left( \pm 0.024 \mathrm{SD}\right.$; Russian prototype). Rescue Balloon ${ }^{\mathrm{TM}}$ showed kinking of the shaft at low bending pressures. The only non-compliant AOB is REBOA Balloon ${ }^{\circledR}$. ER-REBOA ${ }^{\mathrm{TM}}$, Fogarty $^{\circledR}$, LeMaitre $^{\circledR}$, REBOA Balloon ${ }^{\circledR}$, and Rescue Balloon ${ }^{\mathrm{TM}}$ are provided with external length marks to assist blind positioning.

Conclusion In resource-limited settings, a guidewire- and fluoroscopy-free, rather stiff device, such as ER-REBOA ${ }^{\mathrm{TM}}$, Fogarty $^{\circledR}$, and LeMaitre ${ }^{\circledR}$, is warranted. Of these devices, ER-REBOA ${ }^{\mathrm{TM}}$ is the only catheter compatible with seven French sheaths and specifically designed for emergency hemorrhage control. Of the over-the-wire devices, Q50 ${ }^{\circledR}$ has several features that facilitate use and reduce the risk of malplacement or vessel damage.
\end{abstract}

Keyword Aortic occlusion balloon catheter $\cdot$ REBOA $\cdot$ Resuscitation $\cdot$ Stiffness

The opinions or assertions contained herein are the private views of the authors and are not to be construed as official or reflecting the views of the Dutch or U.S. Department of Defense, or Dutch or U.S. governments. Several authors are employees of the Dutch or United States government.

Electronic supplementary material The online version of this article (https://doi.org/10.1007/s00068-020-01382-5) contains supplementary material, which is available to authorized users.

Suzanne M. Vrancken

smvrancken@alrijne.nl

Extended author information available on the last page of the article

\section{Background}

The use of Resuscitative Endovascular Balloon Occlusion of the Aorta (REBOA) as an adjunct for temporary hemorrhage control in patients with exsanguinating torso hemorrhage is increasing. In this technique, an occlusion balloon catheter is inserted, in most of the cases, into the femoral or brachial artery percutaneously or via surgical cut-down. It is then positioned into the aorta. There are two zones of occlusion, depending on the suspected site of injury. Zone I, between the left subclavian artery and the celiac trunk, is for the management of abdominal or retroperitoneal hemorrhage. Zone III occlusion, between the distal renal artery and the aortic bifurcation, is for the management of pelvic, junctional, or proximal lower extremity hemorrhage. Zone II, between the 
celiac trunk and the distal renal artery, is considered as a zone of no occlusion [1-3]. By occluding the aorta above the level of injury, REBOA effectuates temporary distal hemorrhage control and centralizes blood flow. It thereby increases cardiac afterload and central aortic pressure, resulting in sustained perfusion of the brain and heart [4-6].

There are a variety of aortic occlusion balloon catheters (AOB) available with different physical characteristics and specifications. Demands of the AOB differ depending on the indication for REBOA and the circumstances under which REBOA should be performed. The use of REBOA in the fields of trauma and maternal fetal medicine is increasing, thus, consequently, its use in relatively austere settings. Therefore, it is important to select a device that fits the demands of the situation to maximize success and reduce the risk of complications.

The aim of this study is to provide an overview of the commonly used AOB and their specifications, characteristics, bending stiffness and possible advantages and disadvantages in their use to appraise guidance in the choice of an aortic occlusion balloon per medical situation.

\section{Methods}

In this review of available AOB, we assessed 29 AOB for differences, possible advantages and disadvantages based on current literature and experiences with REBOA. Considerations for catheter characteristics will be discussed for different levels of care from resource-limited settings where real-time fluoroscopy is not readily available and other equipment resources may be limited, to more normal levels of care where fluoroscopy and other resources are commonly available.

\section{Exclusion criteria}

AOB from manufacturers or distributors that could not be reached or were not able or willing to provide a sample are excluded from this overview since inclusion would infer missing information that will lead to a disparate comparison.

\section{Specifications}

The AOB are assessed for dimensions of the catheter and balloon, material of the balloon, compatible introducer sheath and guidewire, number of lumina, tip design, radiopacity, features that ease balloon volume control and positioning, and other special features that ease handling of the AOB.

\section{Bending stiffness}

To quantify the bending stiffness (BS) of the AOB, we developed a three-point bending device (MK01-60, Fig. 1). The catheter is placed against two ball bearings and force is applied perpendicular to the longitudinal axis of the catheter midway between the ball bearings. The proximal and distal ends of the catheter can move freely when force is applied to eliminate frictional influence on the measured force. Force is applied and measured at multiple sites of the catheter shaft. Also, since most catheters are packaged rolled up and consequently pre-curved, all catheters are measured in two directions (Fig. 2). The BS of the catheters is measured with and

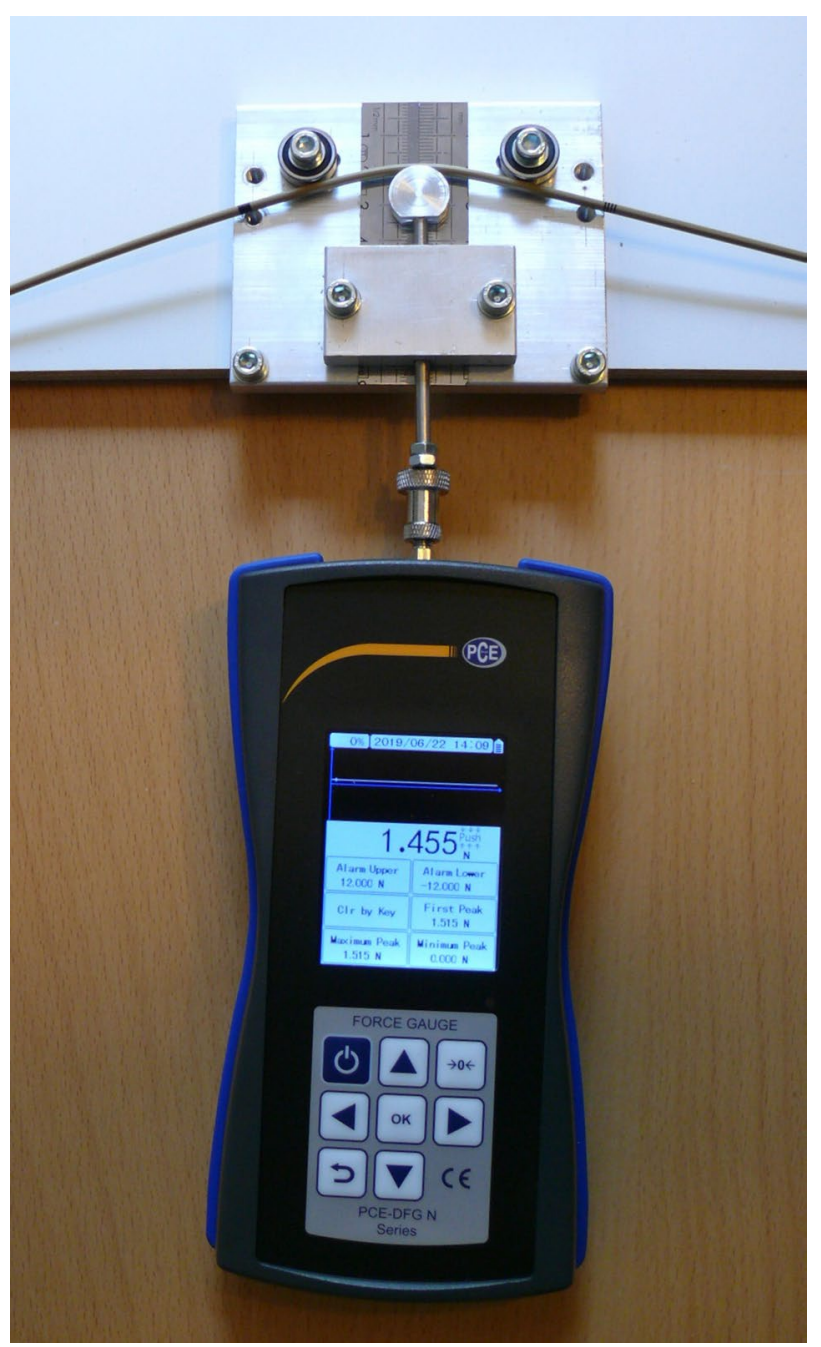

Fig. 1 Three-point bending device (MK01-60) for measuring catheter bending stiffness. The catheter is placed against the two ball bearings and force is applied perpendicular to the longitudinal axis of the catheter midway between the ball bearings. The proximal and distal end of the catheter can move freely when force is applied. The force required to deflect the catheter $10 \mathrm{~mm}$ is measured with a digital force gauge 


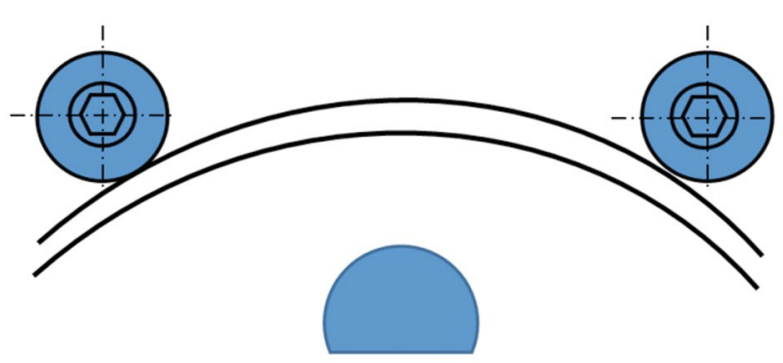

Situation A

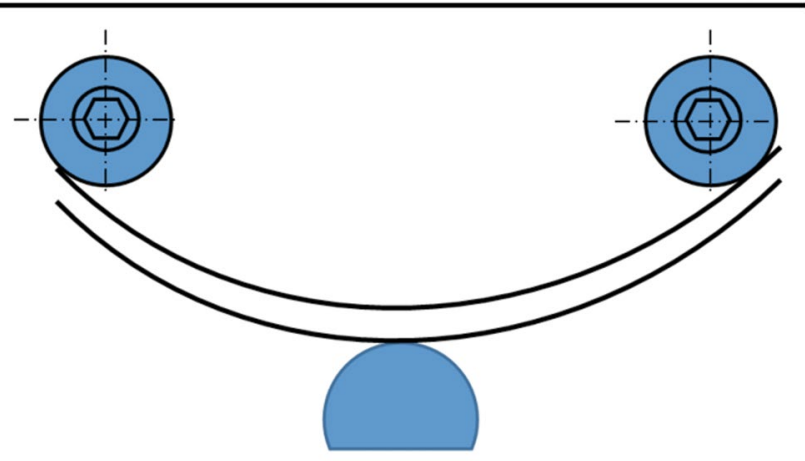

Situation B

Fig. 2 Measurement directions of the catheters. Situation A: force is applied in the direction of the curvature of the catheter. Situation B: force is applied in the opposite direction. The catheter is turned $180^{\circ}$. In case of straight catheters, the same procedure is followed

without guidewire. Catheters without a supplied guidewire are measured with a standard 0.035 inch guidewire (Terumo (Tokyo, Japan) Radifocus ${ }^{\circledR}$ Guidewire M Standard Type $\left.0.035^{\prime \prime}, 150 \mathrm{~cm}\right)$.

The force (Newton) required to deflect the catheter $10 \mathrm{~mm}$ is measured with an ISO calibrated digital force gauge (PCEDFG N 10, PCE Instruments, Meschede, Germany) with a capacity of $0-10 \mathrm{~N}(\mathrm{~N})$, a resolution of $0.005 \mathrm{~N}$ and an accuracy of $0.1 \%$ of full scale. The BS is expressed in $\mathrm{N} / \mathrm{mm}$. Also, the mean stiffness/French is calculated [N/(mm Fr)].

The test bench is validated by repeating $8 / 21(38 \%)$ of the measurements at different times. All measurements are performed at room temperature $\left(21 \pm 0.4{ }^{\circ} \mathrm{C}\right)$.

\section{Results}

Twenty-nine AOB from ten different manufacturers were assessed for variables. Each of these manufacturers provided samples for physical examination. Tables 1 and 2 list the specifications and characteristics of the AOB from these manufacturers. The mean BS and stiffness/French of the obtained catheters is presented in Table 3.
Three AOB were excluded due to various reasons. This involved the ResQ ${ }^{\mathrm{TM}}$ Occlusion Balloon Catheter (QxMédical, Montreal, Quebec, Canada), GORE ${ }^{\circledR}$ Molding \& Occlusion Balloon (W.L. Gore \& Associates, Inc., Flagstaff, AZ, USA), and MIT aortic balloon (Minimally Invasive Technologies, Co. Ltd, Moscow, Russia). Reasons for not being able to include these AOB were: unable to reach the manufacturer, manufacturer was not able or willing to provide a sample or the (new) models were under development.

\section{Aortic occlusion balloon catheters}

\section{Coda ${ }^{\circledR}$ Balloon Catheter (online resource 1)}

Cook Medical, Bloomington, IN, USA, has an assortment of AOB with varying catheter and balloon sizes (Table 1). All catheters are double lumen. The Y-connector has indications for the balloon inflation port ("BALLOON") and wire entry ("DISTAL"). The balloon port is provided with a stopcock for balloon volume control (Table 2).

The Coda-32-LP has a maximum balloon inflation diameter of $32 \mathrm{~mm}$. Its balloon length is $37 \mathrm{~mm}$. The catheter is extremely flexible with a BS of $0.08 \mathrm{~N} / \mathrm{mm}( \pm 0.008 \mathrm{SD})$ without guidewire and $0.11 \mathrm{~N} / \mathrm{mm}( \pm 0.006 \mathrm{SD})$ with guidewire (Table 3).

The Coda- 46 has a maximum balloon inflation diameter of $46 \mathrm{~mm}$ and a balloon length of $38 \mathrm{~mm}$. It is slightly stiffer than the Coda-32-LP. Its BS is $0.12 \mathrm{~N} / \mathrm{mm}( \pm 0.005 \mathrm{SD})$ without guidewire and $0.14 \mathrm{~N} / \mathrm{mm}( \pm 0.010 \mathrm{SD})$ with guidewire (Table 3).

\section{Equalizer $^{\mathrm{TM}}$ Occlusion Balloon Catheter (online resource 2)}

Boston Scientific, Marlborough, MA, USA, offers a wide range of the Equalizer ${ }^{\mathrm{TM}}$ Occlusion Balloon Catheter (Table 1). All catheters have two lumina. The balloon inflation port is marked with "BALLOON" and has a different color than the catheter itself. The central guidewire lumen is marked with "DISTAL" and is shorter than the balloon inflation port. The central lumen can also be used for infusion of contrast medium or medication. Both the balloon port and the central port have a luer-lock connector. There are no stopcocks pre-attached. The 40-mm balloon variants are not suitable for an introducer sheath, but have to be inserted bare via surgical cut-down. There are no length markings on the catheter shaft. According to the instructions for use (IFU), the catheter is designed for temporary vessel occlusion in various applications, including in patients requiring emergency control of hemorrhage (Table 2). The catheter's BS is $0.13 \mathrm{~N} / \mathrm{mm}( \pm 0.032 \mathrm{SD})$ without guidewire and $0.16 \mathrm{~N} /$ $\mathrm{mm}( \pm 0.025 \mathrm{SD})$ with guidewire (Table 3$)$. The Equalizer ${ }^{\mathrm{TM}}$ Occlusion Balloon Catheters are supplied with a syringe. 
Table 1 Specifications of aortic occlusion balloon catheters for REBOA

\begin{tabular}{|c|c|c|c|c|c|c|c|c|}
\hline $\begin{array}{l}\text { Aortic occlusion } \\
\text { balloon }\end{array}$ & $\begin{array}{l}\text { Max infla- } \\
\text { tion } \varnothing \\
{[\mathrm{mm}]}\end{array}$ & $\begin{array}{l}\text { Max inflation } \\
\text { volume [mL] }\end{array}$ & $\begin{array}{l}\text { Catheter } \varnothing \\
{[\mathrm{Fr}]}\end{array}$ & $\begin{array}{l}\text { Required intro- } \\
\text { ducer sheath }\end{array}$ & $\begin{array}{l}\text { Compat- } \\
\text { ible GW } \varnothing \\
{[\text { inch] }}\end{array}$ & Length $[\mathrm{cm}]$ & Lumina & Tip \\
\hline $\begin{array}{l}\text { Coda }^{\circledR} 2-9.0-35- \\
\text { 100-32, Cook } \\
\text { Medical }\end{array}$ & 32 & 30 & 9 & $12 \mathrm{Fr}$ & 0.035 & 100 & 2 & Tapered flexible tip \\
\hline $\begin{array}{l}\text { Coda }^{\circledR} 2-9.0-35- \\
\text { 120-32, Cook } \\
\text { Medical }\end{array}$ & 32 & 30 & 9 & $12 \mathrm{Fr}$ & 0.035 & 120 & 2 & Tapered flexible tip \\
\hline $\begin{array}{l}\text { Coda }^{\circledR} 2-10-35- \\
\text { 140-46, Cook } \\
\text { Medical }\end{array}$ & 46 & 60 & 10 & $14 \mathrm{Fr}$ & 0.035 & 140 & 2 & Tapered flexible tip \\
\hline $\begin{array}{l}\text { Equalizer }^{\mathrm{TM}} \text { Occlu- } \\
\text { sion Balloon } \\
\text { Catheter 17-105, } \\
\text { Boston Scientific }\end{array}$ & 20 & 4.8 & 7 & $14 \mathrm{Fr}$ & 0.038 & 65 & 2 & Stiff tip \\
\hline $\begin{array}{l}\text { Equalizer }^{\mathrm{TM}} \text { Occlu- } \\
\text { sion Balloon } \\
\text { Catheter 17-107, } \\
\text { Boston Scientific }\end{array}$ & 27 & 10 & 7 & $14 \mathrm{Fr}$ & 0.038 & 65 & 2 & Stiff tip \\
\hline $\begin{array}{l}\text { Equalizer }^{\mathrm{TM}} \text { Occlu- } \\
\text { sion Balloon } \\
\text { Catheter 17-109, } \\
\text { Boston Scientific }\end{array}$ & 33 & 19 & 7 & $16 \mathrm{Fr}$ & 0.038 & 65 & 2 & Stiff tip \\
\hline $\begin{array}{l}\text { Equalizer }^{\mathrm{TM}} \text { Occlu- } \\
\text { sion Balloon } \\
\text { Catheter 17-111, } \\
\text { Boston Scientific }\end{array}$ & 40 & 34.5 & 7 & Via cut-down only & 0.038 & 65 & 2 & Stiff tip \\
\hline $\begin{array}{l}\text { Equalizer }^{\mathrm{TM}} \text { Occlu- } \\
\text { sion Balloon } \\
\text { Catheter 17-106, } \\
\text { Boston Scientific }\end{array}$ & 20 & 4.8 & 7 & $14 \mathrm{Fr}$ & 0.038 & 100 & 2 & Stiff tip \\
\hline $\begin{array}{l}\text { Equalizer }^{\mathrm{TM}} \text { Occlu- } \\
\text { sion Balloon } \\
\text { Catheter 17-108, } \\
\text { Boston Scientific }\end{array}$ & 27 & 10 & 7 & $14 \mathrm{Fr}$ & 0.038 & 100 & 2 & Stiff tip \\
\hline $\begin{array}{l}\text { Equalizer }^{\mathrm{TM}} \text { Occlu- } \\
\text { sion Balloon } \\
\text { Catheter 17-110, } \\
\text { Boston Scientific }\end{array}$ & 33 & 19 & 7 & $16 \mathrm{Fr}$ & 0.038 & 100 & 2 & Stiff tip \\
\hline $\begin{array}{l}\text { Equalizer }^{\mathrm{TM}} \text { Occlu- } \\
\text { sion Balloon } \\
\text { Catheter 17-112, } \\
\text { Boston Scientific }\end{array}$ & 40 & 34.5 & 7 & Via cut-down only & 0.038 & 100 & 2 & Stiff tip \\
\hline $\begin{array}{l}\text { ER-REBOA }{ }^{\mathrm{TM}} \text {, } \\
\text { Prytime Medical } \\
\text { Devices }\end{array}$ & 32 & 24 & 6 & $7 \mathrm{Fr}$ & GW-free & 72 & 2 & Atraumatic P-tip ${ }^{\mathrm{TM}}$ \\
\hline $\begin{array}{l}\text { Fogarty }{ }^{\circledR} \text { Occlu- } \\
\text { sion Cath- } \\
\text { eter 62080814F, } \\
\text { Edwards Lifes- } \\
\text { ciences }\end{array}$ & 28 & 10 & 8 & $14 \mathrm{Fr}$ & GW-free & 80 & 1 & $\begin{array}{l}\text { Soft flexible blunt } \\
\text { tip }\end{array}$ \\
\hline $\begin{array}{l}\text { Fogarty }{ }^{\circledR} \text { Occlu- } \\
\text { sion Cath- } \\
\text { eter } 62080822 \mathrm{~F}, \\
\text { Edwards Lifes- } \\
\text { ciences }\end{array}$ & 45 & 43 & 8 & $22 \mathrm{Fr}$ & GW-free & 80 & 1 & $\begin{array}{l}\text { Soft flexible blunt } \\
\text { tip }\end{array}$ \\
\hline
\end{tabular}


Table 1 (continued)

\begin{tabular}{|c|c|c|c|c|c|c|c|c|}
\hline $\begin{array}{l}\text { Aortic occlusion } \\
\text { balloon }\end{array}$ & $\begin{array}{l}\text { Max infla- } \\
\text { tion } \varnothing \\
{[\mathrm{mm}]}\end{array}$ & $\begin{array}{l}\text { Max inflation } \\
\text { volume [mL] }\end{array}$ & $\begin{array}{l}\text { Catheter } \varnothing \\
{[\mathrm{Fr}]}\end{array}$ & $\begin{array}{l}\text { Required intro- } \\
\text { ducer sheath }\end{array}$ & $\begin{array}{l}\text { Compat- } \\
\text { ible GW } \varnothing \\
\text { [inch] }\end{array}$ & Length $[\mathrm{cm}]$ & Lumina & Tip \\
\hline $\begin{array}{l}\text { LeMaitre }{ }^{\circledR} \\
\text { 2107-80 Aortic } \\
\text { Occlusion Cath- } \\
\text { eter, LeMaitre } \\
\text { Vascular }\end{array}$ & 28 & 15 & 8 & $14 \mathrm{Fr}$ & GW-free & 80 & 1 & $\begin{array}{l}\text { Soft flexible blunt } \\
\text { tip }\end{array}$ \\
\hline $\begin{array}{l}\text { LeMaitre } \\
\text { 2107-81 Aortic } \\
\text { Occlusion Cath- } \\
\text { eter, LeMaitre } \\
\text { Vascular }\end{array}$ & 45 & 50 & 8 & $22 \mathrm{Fr}$ & GW-free & 80 & 1 & $\begin{array}{l}\text { Soft flexible blunt } \\
\text { tip }\end{array}$ \\
\hline $\begin{array}{l}\text { Q50 }{ }^{\circledR} \text { Plus Q50- } \\
\text { 65P, QXMédical } \\
\text { (Merit Medical) }\end{array}$ & 50 & 60 & 8 & $12 \mathrm{Fr}$ & 0.038 & 65 & 3 & Tapered flexible tip \\
\hline $\begin{array}{l}\text { Q50 }{ }^{\circledR} \text { Plus Q50- } \\
\text { 100P, QXMé- } \\
\text { dical (Merit } \\
\text { Medical) }\end{array}$ & 50 & 60 & 8 & $12 \mathrm{Fr}$ & 0.038 & 100 & 3 & Tapered flexible tip \\
\hline $\begin{array}{l}\text { Q50X } \\
\text { 65-X* Q50- } \\
\text { dical (Merit } \\
\text { Medical) }\end{array}$ & 50 & 60 & 8 & $10 \mathrm{Fr}$ & 0.038 & 65 & 3 & Tapered flexible tip \\
\hline $\begin{array}{l}\text { Q50X'Тм Q50- } \\
\text { 100-X*, QXMé- } \\
\text { dical (Merit } \\
\text { Medical) }\end{array}$ & 50 & 60 & 8 & $10 \mathrm{Fr}$ & 0.038 & 100 & 3 & Tapered flexible tip \\
\hline $\begin{array}{l}\text { REBOA Balloon }{ }^{\circledR} \\
\text { 15, REBOA } \\
\text { Medical AS }\end{array}$ & 15 & 8 & 6 & $6 \mathrm{Fr}$ & 0.035 & $30 / 50 / 70$ & 2 & Stiff tapered tip \\
\hline $\begin{array}{l}\text { REBOA Balloon }^{\circledR} \\
\text { 20, REBOA } \\
\text { Medical AS }\end{array}$ & 20 & 15 & 7 & $7 \mathrm{Fr}$ & 0.035 & $30 / 50 / 70$ & 2 & Stiff tapered tip \\
\hline $\begin{array}{l}\text { Reliant }{ }^{\mathrm{TM}} \text { Stent } \\
\text { Graft Bal- } \\
\text { loon Catheter, } \\
\text { Medtronic }\end{array}$ & 46 & 60 & 8 & $12 \mathrm{Fr}$ & 0.038 & 100 & 2 & Stiff tapered tip \\
\hline $\begin{array}{l}\text { Rescue balloon }{ }^{\mathrm{TM}} \\
\text { OBS-01A, Tokai } \\
\text { Medical Products }\end{array}$ & 40 & 40 & 7 & $7 \mathrm{Fr}$ & 0.025 & 100 & 2 & Flexible tip \\
\hline $\begin{array}{l}\text { Russian prototype } \\
\text { AOB, Minimally } \\
\text { Invasive Tech- } \\
\text { nologies }\end{array}$ & 40 & 45 & 6 & $10 \mathrm{Fr}$ & GW-free & 100 & 1 & J-tip \\
\hline
\end{tabular}

$A O B$ aortic occlusion balloon, $c m$ centimeters, $F r$ French, $G W$ guidewire, $m L$ milliliters, $m m$ millimeter

*Currently only available in the USA

\section{ER-REBOA $^{\mathrm{TM}}$ (online resource 3 )}

The ER-REBOA ${ }^{\mathrm{TM}}$ from Prytime Medical Devices, Boerne, TX, USA, is a 6-Fr, guidewire-free, double-lumen catheter (Table 1). The catheter shaft uses a concentric tube-in-tube design with inner elastic nitinol tube and outer plastic tube [7]. The inner lumen is used for arterial pressure monitoring with a pressure port distal from the balloon; the outer lumen is for balloon inflation. The ER-REBOA ${ }^{\mathrm{TM}}$ has an atraumatic
P-tip ${ }^{\mathrm{TM}}$ designed to resist accidental entry into aortic side branches [7]. A peel-away sheath facilitates insertion of the catheter. The maximum balloon inflation diameter is $32 \mathrm{~mm}$ and the length of the balloon is $48 \mathrm{~mm}$. The catheter shaft has external length marks every centimeter and indications of every $5 \mathrm{~cm}$ to facilitate placement in situations without fluoroscopy (Table 2). Both the balloon inflation port and the arterial pressure port of the Y-connector have a flexible extension with stopcock to facilitate balloon volume control 
Table 2 Characteristics and features of aortic occlusion balloon catheters for REBOA

\begin{tabular}{|c|c|c|c|c|}
\hline Aortic occlusion balloon & Material & Features & Indications & Price* \\
\hline $\begin{array}{l}\text { Coda }{ }^{\circledR} \text { Balloon Catheter, Cook } \\
\text { Medical }\end{array}$ & Compliant polyurethane & $\begin{array}{l}\text { Radiopaque markers at the bal- } \\
\text { loon to assist with positioning } \\
\text { under fluoroscopy } \\
\text { Distinctive balloon port and } \\
\text { central lumen port } \\
\text { Stopcock to facilitate balloon } \\
\text { volume control }\end{array}$ & $\begin{array}{l}\text { Temporary occlusion of large } \\
\text { vessels } \\
\text { To expand vascular prostheses }\end{array}$ & $€ 450$ \\
\hline $\begin{array}{l}\text { Equalizer }{ }^{\mathrm{TM}} \text { Occlusion Balloon } \\
\text { Catheter, Boston Scientific }\end{array}$ & Compliant natural rubber latex & $\begin{array}{l}\text { Radiopaque catheter shaft } \\
\text { Radiopaque markers at the bal- } \\
\text { loon to assist with positioning } \\
\text { under fluoroscopy } \\
\text { Distinctive balloon port and } \\
\text { central lumen port } \\
\text { Supplied with a syringe }\end{array}$ & $\begin{array}{l}\text { Temporary occlusion of large } \\
\text { vessels } \\
\text { Including emergency control of } \\
\text { hemorrhage }\end{array}$ & $€ 100$ \\
\hline $\begin{array}{l}\text { ER-REBOA }^{\mathrm{TM}} \text {, Prytime Medical } \\
\text { Devices }\end{array}$ & $\begin{array}{l}\text { Compliant thermoplastic } \\
\text { elastomer }\end{array}$ & $\begin{array}{l}\text { Guidewire-free } \\
\text { External length marks every } \\
\text { cm for positioning without } \\
\text { fluoroscopy } \\
\text { Radiopaque catheter shaft } \\
\text { Radiopaque markers at the bal- } \\
\text { loon to assist with positioning } \\
\text { under fluoroscopy } \\
\text { Pre-loaded peel-away sheath to } \\
\text { ease insertion of the catheter's } \\
\text { P-tip } \\
\text { Built-in arterial pressure lumen } \\
\text { Distinctive balloon port and } \\
\text { central lumen port } \\
\text { Stopcock to facilitate balloon } \\
\text { volume control } \\
\text { Available with convenience sets } \\
\text { and access kits }\end{array}$ & $\begin{array}{l}\text { Temporary occlusion of large } \\
\text { vessels } \\
\text { Monitoring of blood pressure } \\
\text { Including emergency control of } \\
\text { hemorrhage }\end{array}$ & $€ 1950$ \\
\hline $\begin{array}{l}\text { Fogarty }{ }^{\circledR} \text { Occlusion Catheter, } \\
\text { Edwards Lifesciences }\end{array}$ & Compliant natural rubber latex & $\begin{array}{l}\text { Guidewire-free } \\
\text { Length marks every } 10 \mathrm{~cm} \text { to } \\
\text { assist positioning without } \\
\text { fluoroscopy } \\
\text { Radiopaque catheter shaft } \\
\text { Gate valve to facilitate balloon } \\
\text { volume control }\end{array}$ & $\begin{array}{l}\text { Temporary occlusion of large } \\
\text { vessels }\end{array}$ & $€ 155$ \\
\hline $\begin{array}{l}\text { LeMaitre }^{\circledR} \text { Aortic Occlusion } \\
\text { Catheter, LeMaitre Vascular }\end{array}$ & Compliant natural rubber latex & $\begin{array}{l}\text { Guidewire-free } \\
\text { Length marks every } 10 \mathrm{~cm} \text { to } \\
\text { assist positioning without } \\
\text { fluoroscopy } \\
\text { Radiopaque catheter shaft } \\
\text { Stopcock to facilitate balloon } \\
\text { volume control }\end{array}$ & $\begin{array}{l}\text { Temporary occlusion of large } \\
\text { vessels }\end{array}$ & $€ 135$ \\
\hline $\begin{array}{l}\text { Q50 }{ }^{\circledR} \text { Stent Graft Balloon } \\
\text { Catheter, QXMédical (Merit } \\
\text { Medical) }\end{array}$ & Compliant polyurethane & $\begin{array}{l}\text { Three lumina to allow quick } \\
\text { inflation and deflation of the } \\
\text { balloon } \\
\text { Radiopaque markers at the bal- } \\
\text { loon to assist with positioning } \\
\text { under fluoroscopy } \\
\text { Stopcock to facilitate balloon } \\
\text { volume control }\end{array}$ & $\begin{array}{l}\text { Temporary occlusion of large } \\
\text { vessels } \\
\text { To expand vascular prostheses }\end{array}$ & $€ 400$ \\
\hline
\end{tabular}


Table 2 (continued)

\begin{tabular}{|c|c|c|c|c|}
\hline Aortic occlusion balloon & Material & Features & Indications & Price* \\
\hline $\begin{array}{l}\text { REBOA Balloon }{ }^{\circledR}, \text { REBOA } \\
\text { Medical AS }\end{array}$ & $\begin{array}{l}\text { Non-compliant thermoplastic } \\
\text { elastomer }\end{array}$ & $\begin{array}{l}\text { One or two length marks on the } \\
\text { catheter shaft to assist posi- } \\
\text { tioning without fluoroscopy } \\
\text { Radiopaque markers at the bal- } \\
\text { loon to assist with positioning } \\
\text { under fluoroscopy } \\
\text { Distinctive balloon port and } \\
\text { central lumen port } \\
\text { Available with a complete } \\
\text { kit containing all materials } \\
\text { needed for REBOA }\end{array}$ & $\begin{array}{l}\text { Temporary occlusion of the } \\
\text { aorta }\end{array}$ & $€ 1350^{\dagger}$ \\
\hline $\begin{array}{l}\text { Reliant }^{\mathrm{TM}} \text { Stent Graft Balloon } \\
\text { Catheter, Medtronic }\end{array}$ & Compliant polyurethane & $\begin{array}{l}\text { Radiopaque markers at the bal- } \\
\text { loon to assist with positioning } \\
\text { under fluoroscopy } \\
\text { Stopcock to facilitate balloon } \\
\text { volume control }\end{array}$ & $\begin{array}{l}\text { Temporary occlusion of large } \\
\text { vessels } \\
\text { To expand vascular prostheses } \\
\text { or assist in the expansion of } \\
\text { self-expanding stent grafts }\end{array}$ & $€ 180$ \\
\hline $\begin{array}{l}\text { Rescue balloon }{ }^{\mathrm{TM}} \text {, Tokai Medi- } \\
\text { cal Products }\end{array}$ & Compliant polyurethane & $\begin{array}{l}\text { Length marks every } 5 \mathrm{~cm} \text { to } \\
\text { assist positioning without } \\
\text { fluoroscopy } \\
\text { Radiopaque markers at the bal- } \\
\text { loon to assist with positioning } \\
\text { under fluoroscopy } \\
\text { Stopcock to facilitate balloon } \\
\text { volume control }\end{array}$ & $\begin{array}{l}\text { Temporary occlusion of large } \\
\text { vessels } \\
\text { Including emergency control of } \\
\text { hemorrhage }\end{array}$ & NA \\
\hline $\begin{array}{l}\text { Russian prototype AOB, Mini- } \\
\text { mally Invasive Technologies }\end{array}$ & Latex & $\begin{array}{l}\text { Guidewire-free } \\
\text { Radiopaque catheter shaft } \\
\text { Stopcock to facilitate balloon } \\
\text { volume control } \\
\text { Rubber ring that can indicate } \\
\text { migration }\end{array}$ & $\begin{array}{l}\text { Temporary occlusion of large } \\
\text { vessels }\end{array}$ & NA \\
\hline
\end{tabular}

$A O B$ aortic occlusion balloon, $N A$ price information not yet available

*Approximate catalog price. Prices may vary based on regional differences, taxes, price agreements and changes over time

${ }^{\dagger}$ Price for complete procedure kit. The balloon catheter is not sold separately

and fluid control of the arterial line. The balloon arm has a white stopcock and indicates "BAL". The arterial pressure port comes with a red stopcock and indicates "ART". The BS of the catheter is $0.43 \mathrm{~N} / \mathrm{mm}( \pm 0.013 \mathrm{SD})$ (Table 3$)$.

The ER-REBOA ${ }^{\mathrm{TM}}$ is the most expensive catheter (Table 2). It is specifically designed for temporary hemorrhage control and resuscitation support in emergency settings. Convenience sets and access kits are available containing materials needed for arterial access, sheath placement and fixation of the catheter.

\section{Fogarty ${ }^{\circledR}$ Occlusion Catheter (online resource 4)}

The Fogarty ${ }^{\circledR}$ Occlusion Catheter from Edwards Lifesciences, Irvine, CA, USA, comes in two models suitable for aortic occlusion (Table 1). Both catheters are singlelumen, guidewire-free catheters with a blunt flexible tip. The catheters come with a removable stylet that increases body stiffness during placement of the catheter [BS $0.25 \pm 0.011 \mathrm{~N} / \mathrm{mm}$ with stylet versus $0.12 \pm 0.007 \mathrm{~N} / \mathrm{mm}$ without stylet (Table 3)]. Marker bands assisting positioning without fluoroscopy are located on the catheter shaft every $10 \mathrm{~cm}$, decreasing in number and width towards the balloon (Table 2). The catheter shaft itself is radiopaque. It does not have distinct radiopaque marker bands at the balloon. A gate valve with luer-lock connector is attached to the proximal end of the catheter (the inflation port). Closing the gate valve prevents the balloon from deflation. The catheters are supplied in a straight tube and are therefore not pre-curved.

\section{LeMaitre ${ }^{\circledast}$ Aortic Occlusion Catheter (online resource 5)}

LeMaitre ${ }^{\circledR}$ Vascular, Burlington, MA, USA, offers two types of AOB suitable for REBOA (Table 1). Both models are guidewire-free, single-lumen catheters with an atraumatic flexible tip, and a two-way stopcock attached to the single lumen to maintain balloon inflation volume. Unique length markings are located on the catheter shaft every $10 \mathrm{~cm}$ to assist positioning without fluoroscopy (Table 2). There are no radiopaque marker bands located at the balloon; however, 
Table 3 Bending stiffness of aortic occlusion balloon catheters for REBOA

\begin{tabular}{|c|c|c|c|c|c|c|c|}
\hline $\begin{array}{l}\text { Aortic occlusion } \\
\text { balloon }\end{array}$ & $\begin{array}{l}\text { Overall mean } \\
\mathrm{BS} \text { w/o GW }[\mathrm{N} / \\
\mathrm{mm}]( \pm \mathrm{SD})\end{array}$ & $\begin{array}{l}\text { Mean BS/Fr } \\
\text { w/o GW [N/ } \\
(\mathrm{mm} \mathrm{Fr})]\end{array}$ & $\begin{array}{l}\text { Overall mean } \\
\mathrm{BS} \text { w/ GW }[\mathrm{N} / \\
\mathrm{mm}]( \pm \mathrm{SD})\end{array}$ & $\begin{array}{l}\text { Mean BS/Fr w/ } \\
\text { GW }[\mathrm{N} /(\mathrm{mm} \\
\mathrm{Fr})]\end{array}$ & $\begin{array}{l}\text { Overall mean } \\
\mathrm{BS} \text { w/ stylet }[\mathrm{N} / \\
\mathrm{mm}]( \pm \mathrm{SD})\end{array}$ & $\begin{array}{l}\text { Mean BS/Fr w/ } \\
\text { stylet }[\mathrm{N} /(\mathrm{mm} \\
\mathrm{Fr})]\end{array}$ & Guidewire \\
\hline $\begin{array}{l}\text { Coda-2-9.0-35- } \\
120-32\end{array}$ & $0.08( \pm 0.008)$ & 0.009 & $0.11( \pm 0.006)$ & 0.012 & $\mathrm{n} / \mathrm{a}$ & $\mathrm{n} / \mathrm{a}$ & $\begin{array}{l}\text { Terumo } \\
\text { Radifocus }{ }^{\circledR} \\
\text { Guidewire M } \\
\text { Standard Type } \\
0.035^{\prime \prime}\end{array}$ \\
\hline $\begin{array}{l}\text { Coda-2-10-35- } \\
140-46\end{array}$ & $0.12( \pm 0.005)$ & 0.012 & $0.14( \pm 0.010)$ & 0.014 & $\mathrm{n} / \mathrm{a}$ & $\mathrm{n} / \mathrm{a}$ & $\begin{array}{l}\text { Terumo } \\
\text { Radifocus }{ }^{\circledR} \\
\text { Guidewire M } \\
\text { Standard Type } \\
0.035^{\prime \prime}\end{array}$ \\
\hline $\begin{array}{c}\text { Equalizer }^{\mathrm{TM}} \\
17-109\end{array}$ & $0.13( \pm 0.032)$ & 0.019 & $0.16( \pm 0.025)$ & 0.023 & $\mathrm{n} / \mathrm{a}$ & $\mathrm{n} / \mathrm{a}$ & $\begin{array}{l}\text { Terumo } \\
\text { Radifocus }{ }^{\circledR} \\
\text { Guidewire M } \\
\text { Standard Type } \\
0.035^{\prime \prime}\end{array}$ \\
\hline ER-REBOA $^{\mathrm{TM}}$ & $0.43( \pm 0.013)$ & 0.071 & $\mathrm{n} / \mathrm{a}$ & $\mathrm{n} / \mathrm{a}$ & $\mathrm{n} / \mathrm{a}$ & $\mathrm{n} / \mathrm{a}$ & $\mathrm{n} / \mathrm{a}$ \\
\hline $\begin{array}{l}\text { Fogarty }{ }^{\circledR} \\
\quad 62080814 \mathrm{~F}\end{array}$ & $0.12( \pm 0.007)$ & 0.015 & $\mathrm{n} / \mathrm{a}$ & $\mathrm{n} / \mathrm{a}$ & $0.25( \pm 0.011)$ & 0.031 & $\mathrm{n} / \mathrm{a}$ \\
\hline $\begin{array}{c}\text { LeMaitre }^{\circledR} \\
2107-80\end{array}$ & $0.33( \pm 0.013)$ & 0.041 & $\mathrm{n} / \mathrm{a}$ & $\mathrm{n} / \mathrm{a}$ & $\mathrm{n} / \mathrm{a}$ & $\mathrm{n} / \mathrm{a}$ & $\mathrm{n} / \mathrm{a}$ \\
\hline $\begin{array}{c}\text { LeMaitre }^{\circledR} \\
2107-81\end{array}$ & $0.33( \pm 0.017)$ & 0.042 & $\mathrm{n} / \mathrm{a}$ & $\mathrm{n} / \mathrm{a}$ & $\mathrm{n} / \mathrm{a}$ & $\mathrm{n} / \mathrm{a}$ & $\mathrm{n} / \mathrm{a}$ \\
\hline $\begin{array}{l}\text { Q50 } \\
\text { 65P Plus Q50- }\end{array}$ & $0.30( \pm 0.061)$ & 0.037 & $0.34( \pm 0.043)$ & 0.042 & $\mathrm{n} / \mathrm{a}$ & $\mathrm{n} / \mathrm{a}$ & $\begin{array}{l}\text { Terumo } \\
\text { Radifocus }{ }^{\circledR} \\
\text { Guidewire M } \\
\text { Standard Type } \\
0.035^{\prime \prime}\end{array}$ \\
\hline $\begin{array}{l}\text { REBOA } \\
\text { Balloon }{ }^{\circledR} 20\end{array}$ & $0.17( \pm 0.036)$ & 0.024 & $0.30( \pm 0.040)$ & 0.042 & $\mathrm{n} / \mathrm{a}$ & $\mathrm{n} / \mathrm{a}$ & $\begin{array}{l}\text { SP Medical } \\
\text { Accoat Guide } \\
\text { Wire Seldinger } \\
\text { Extra stiff, } \\
0.035^{\prime \prime}\end{array}$ \\
\hline Reliant $^{\mathrm{TM}}$ & $0.11( \pm 0.016)$ & 0.014 & $0.14( \pm 0.017)$ & 0.017 & $\mathrm{n} / \mathrm{a}$ & $\mathrm{n} / \mathrm{a}$ & $\begin{array}{l}\text { Terumo } \\
\text { Radifocus }{ }^{\circledR} \\
\text { Guidewire M } \\
\text { Standard Type } \\
0.035^{\prime \prime}\end{array}$ \\
\hline $\begin{array}{l}\text { Rescue bal- } \\
\text { loon }^{\mathrm{TM}}\end{array}$ & $0.23( \pm 0.048)$ & 0.032 & $0.26( \pm 0.051)$ & 0.038 & $0.47( \pm 0.036)$ & 0.067 & $\begin{array}{l}\text { Hydrophilic GW } \\
0.025^{\prime \prime}\end{array}$ \\
\hline $\begin{array}{l}\text { Russian proto- } \\
\text { type }\end{array}$ & $0.72( \pm 0.024)$ & 0.119 & $\mathrm{n} / \mathrm{a}$ & $\mathrm{n} / \mathrm{a}$ & $\mathrm{n} / \mathrm{a}$ & $\mathrm{n} / \mathrm{a}$ & $\mathrm{n} / \mathrm{a}$ \\
\hline
\end{tabular}

$B S$ bending stiffness, $F r$ French, $G W$ guidewire, $m m$ millimeter, $N$ Newton, $n / a$ not applicable, $S D$ standard deviation, $w /$ with, $w / o$ without

the catheter shaft itself is radiopaque. The catheters are not pre-curved since they are supplied in a straight tube.

The LeMaitre ${ }^{\circledR} 2107-80$ has a maximum balloon inflation diameter of $28 \mathrm{~mm}$ and is compatible with a 14-Fr introducer sheath. The balloon length is approximately $20 \mathrm{~mm}$. BS is $0.33 \mathrm{~N} / \mathrm{mm}( \pm 0.013 \mathrm{SD})$ (Table 3$)$.

The LeMaitre ${ }^{\circledR} 2107-81$ has a maximum inflation diameter of $45 \mathrm{~mm}$ and is compatible with a $22-\mathrm{Fr}$ introducer sheath. Its balloon length is $24 \mathrm{~mm}$. It has a BS of $0.33 \mathrm{~N} /$ $\mathrm{mm}( \pm 0.017 \mathrm{SD})$ (Table 3).

\section{Q50 ${ }^{\circledR}$ Stent Graft Balloon Catheter (online resource 6)}

QXMédical, Montreal, Quebec, Canada, manufactures four types of the Q50 ${ }^{\circledR}$ Stent Graft Balloon Catheter that are either compatible with a 12-Fr introducer sheath or a 10-Fr sheath (Table 1). The difference in introducer sheath compatibility is due to a different balloon wrapping of the Q50 ${ }^{\circledR}$ PLUS and the Q50X ${ }^{\mathrm{TM}}$. With a maximum balloon diameter of $50 \mathrm{~mm}$, the $\mathrm{Q} 50^{\circledR}$ is the largest occlusion balloon. It has a length of approximately $40 \mathrm{~mm}$. The Q50 ${ }^{\circledR}$ Stent Graft 
Balloon Catheters are the only AOB with three lumina. It has two balloon inflation lumina to allow quick inflation and deflation of the balloon. The third lumen is intended for a guidewire. The balloon inflation port has an extension tube with a stopcock to prevent balloon deflation (Table 2). Q50 ${ }^{\circledR}$ distributor Merit Medical (South Jordan, UT, USA) offers a lockable syringe (VacLok ${ }^{\circledR}$ Negative Pressure Syringe) to facilitate balloon volume control. BS is $0.30 \mathrm{~N} / \mathrm{mm}( \pm 0.061$ $\mathrm{SD})$ without guidewire and $0.34 \mathrm{~N} / \mathrm{mm}( \pm 0.043 \mathrm{SD})$ with guidewire (Table 3).

\section{REBOA Balloon $^{\oplus}$ (online resource 7)}

REBOA Medical AS, Norway, offers a broad range of the REBOA Balloon ${ }^{\circledR}$ catheter (Table 1). It is the only AOB with a non-compliant balloon. It consists of a thermoplastic elastomer (DEHP free and latex free). The maximum diameter of the balloon with standard injection volume avoids aortic wall rupture since over-inflation is not possible. However, it does impose a risk of rupturing the aorta in patients with a smaller aortic diameter. In situations where the aortic diameter is in excess of the occlusion balloon diameter, the balloon will not provide occlusion or hemorrhage control.

The REBOA Medical AS balloon catheters are available with a 15-mm (6-Fr) or 20-mm (7-Fr) balloon diameter The catheters can only be ordered as a complete kit containing all materials needed for REBOA, including a guidewire (SP Medical Accoat Guide Wire Seldinger Extra stiff, J3 mm tip, PTFE coated, 150-cm length, 0.035-inch diameter). The catheters have a coaxial double-lumen design. The balloon inflation port is marked with "BALLOON" and is longer, and has a different color than the guidewire lumen (Table 2). According to the IFU, the catheters can be placed without fluoroscopic guidance. To aid positioning without fluoroscopy, the 50-cm catheters have a shaft marking at $30 \mathrm{~cm}$ from the distal tip. The $70-\mathrm{cm}$ catheters have single line marking at $30 \mathrm{~cm}$ from the distal tip and a double line at $50 \mathrm{~cm}$ from the distal tip. The BS of the 7-Fr catheter is $0.17 \mathrm{~N} / \mathrm{mm}( \pm 0.036 \mathrm{SD})$ without guidewire and $0.30 \mathrm{~N} / \mathrm{mm}$ $( \pm 0.040 \mathrm{SD})$ with guidewire (Table 3$)$.

\section{Reliant $^{\mathrm{Tm}}$ Stent Graft Balloon Catheter (online resource 8)}

The Reliant ${ }^{\mathrm{TM}}$ Stent Graft Balloon Catheter from Medtronic, Minneapolis, MN, USA, is a flexible double-lumen catheter with a $\mathrm{BS}$ of $0.11 \mathrm{~N} / \mathrm{mm}( \pm 0.016 \mathrm{SD})$ without guidewire and $0.14 \mathrm{~N} / \mathrm{mm}( \pm 0.017 \mathrm{SD})$ with guidewire (Table 3$)$. The balloon has a maximum inflation diameter of $46 \mathrm{~mm}$ and a length of approximately $37 \mathrm{~mm}$ (Table 1 ). There are no length markings on the catheter shaft. The balloon inflation port of the Y-connector has a flexible extension with a threeway stopcock to assist in balloon volume control (Table 2).

\section{Rescue Balloon $^{\mathrm{Tm}}$ (online resource 9)}

The Tokai Rescue Balloon ${ }^{\mathrm{TM}}$ OBS-01A from Tokai Medical Products, Aichi, Japan, is a small-sized, double-lumen 7-Fr catheter (Table 1). It can be used with a 0.025-inch guidewire that is provided with the catheter. After balloon positioning, the guidewire can be removed and a stylet can be inserted to provide additional catheter stiffness [BS $0.47 \pm 0.036 \mathrm{~N} / \mathrm{mm}$ with stylet versus $0.26 \pm 0.051 \mathrm{~N} / \mathrm{mm}$ with guidewire (Table 3)]. The BS of the catheter is $0.23 \mathrm{~N} /$ $\mathrm{mm}( \pm 0.048 \mathrm{SD})$. Length marks that assist with positioning without fluoroscopy are located on the catheter shaft every $5 \mathrm{~cm}$ (Table 2). A double mark is located $55 \mathrm{~cm}$ from the tip of the catheter. The balloon inflation port has a flexible extension with a stopcock to prevent deflation of the balloon during the procedure.

The catheter tends to kink and when kinked, it remains plastically deformed. Careful handling of the catheter to prevent kinking prior to insertion is important.

\section{Russian prototype aortic occlusion balloon (online resource 10)}

The Russian prototype AOB from Minimally Invasive Technologies, Co. Ltd, Moscow, Russia, is a small-sized 6-Fr catheter (Table 1). The deflated balloon requires a $10-\mathrm{Fr}$ sheath. Despite the small diameter, it is the stiffest catheter with a BS of $0.72 \mathrm{~N} / \mathrm{mm}( \pm 0.024 \mathrm{SD})$ (Table 3$)$. The maximum inflation diameter of the balloon is $40 \mathrm{~mm}$ and its length is approximately $34 \mathrm{~mm}$. The catheter has a built-in J-tip guidewire which cannot be removed from the catheter. It also has a rubber stop ring that can be used to indicate initial introduction depth and possible subsequent migration of the catheter (Table 2). There are no length markings on the catheter shaft. The transition from catheter shaft to guidewire tip is visible with fluoroscopy.

\section{Discussion}

There is a wide variety of AOB available and specifications, and characteristics are diverse. With the increasing use of REBOA in the field of trauma, maternal fetal medicine and iatrogenic surgical hemorrhage, it is important to choose a device that fits the demands for the best success rate for a patient in need of urgent hemorrhage control. However, there is no consensus on the desired specific features of the AOB. This article provides an overview of the currently available AOB with their specifications, characteristics and bending stiffness, and possible advantages and disadvantages in their use after extensive review of the catheters. Cautious recommendations for selecting an AOB are done based on this review, current literature and experience with REBOA. 
Only three AOB, the Equalizer ${ }^{\mathrm{TM}}$, ER-REBOA ${ }^{\mathrm{TM}}$, and Rescue balloon ${ }^{\mathrm{TM}}$ have specifically indicated in their IFU that it is intended for use in patients with massive bleeding. Suitability for partial aortic occlusion is not described in any of the IFUs. All catheters are small caliber catheters. Required introducer sheath size and the catheters BS, however, vary widely. We found that the guidewire-free devices are generally stiffer than the over-the-wire catheters and a standard guidewire only adds minimal extra stiffness. Catheter stiffness, including a stiff catheter tip, might increase the risk of dissection or rupture of the aorta during placement [8]. On the other hand, Onishi et al. described a case of a loop formation of an AOB in the aorta presumably due to a more flexible small caliber catheter [9]. Borger van der Burg et al. also demonstrates an association between catheter stiffness and migration of the aortic occlusion balloon [10]. In their study, minor primary migration during insufflation is observed in all devices. Only the Cook Coda ${ }^{\circledR}$ Balloon Catheter and the Russian prototype AOB showed subsequent migration under higher pressures. Comparing these findings with our results, we can conclude that the most flexible and the stiffest catheters migrated while fully inflated, suggesting there is an optimum in catheter stiffness regarding the risk of balloon migration. The stiff catheter (Russian prototype AOB, BS $0.72 \mathrm{~N} / \mathrm{mm}( \pm 0.024 \mathrm{SD}))$ showed outward migration at the sheath; while, migration of the highly flexible catheters $\left(9 \mathrm{Fr} \mathrm{Coda}^{\circledR}\right.$ with BS of $0.08 \mathrm{~N} / \mathrm{mm}( \pm 0.008 \mathrm{SD})$ and 10-Fr Coda ${ }^{\circledR}$ with BS of $0.012 \mathrm{~N} / \mathrm{mm}( \pm 0.005 \mathrm{SD})$ ) was due to kinking of the catheter in the aorta which was not visible at the sheath. Theoretically, outward migration or kinking of the catheter is also possible in patients in whom restoration of blood pressure leads to an increase in aortic diameter and consequently loss of contact surface between the balloon and aortic wall, while downward pressure on the catheter is increasing. By general rules, the axial force a catheter can resist is limited by the buckling force. The buckling force depends on the BS as well as the aspect ratio (relation between length, area, and moment of inertia) of the catheter. A catheter with a high BS is more resistant to kinking due to in vivo axial pressure. However, the buckling force of most catheters will be negligible due to the large aspect ratio. The flexible Reliant ${ }^{\mathrm{TM}}$ catheter (BS 0.11 $( \pm 0.016 \mathrm{SD}))$ did not show secondary migration in the study of Borger van der Burg et al. This supports the theory that frictional force between the balloon and aortic wall is of importance in preventing migration or kinking of the catheter. Despite the observed migration, all catheters achieved total aortic occlusion and can be used for REBOA. However, in vivo kinking of the catheter shaft can lead to an improper occlusion location when fluoroscopy is not available to confirm the position of the balloon [9-11]. In resource-limited settings where real-time fluoroscopy is not readily available, such as the obstetrics delivery room, intensive care unit, remote areas (such as military treatment facilities) or out-of-hospital REBOA, catheters with medium to high stiffness are, therefore, a safer option. These include the ERREBOA $^{\mathrm{TM}}$, LeMaitre ${ }^{\circledR}$, or Fogarty ${ }^{\circledR}$ with stylet. Obtaining plain radiographs directly before and after balloon inflation to confirm balloon position can be an alternative for fluoroscopic guidance. However, real-time fluoroscopic guidance is always preferred to monitor possible migration or overinflation with consequent rupture of the balloon or aorta.

In environments with limited equipment resources or supporting staff that are not familiar with endovascular materials, the complete procedure kits from REBOA Medical AS (REBOA Balloon ${ }^{\circledR}$ ) or Prytime Medical Devices (ERREBOA $^{\mathrm{TM}}$ ) are beneficial. When considering health care costs as limited resource barrier, the ER-REBOA ${ }^{\mathrm{TM}}$ (approximately 1950 euro) can be a barrier, especially in middle- to low-income countries. To explore the need for REBOA in such countries, we are currently preparing a study into noncompressible truncal hemorrhage in the Republic of South Africa (RSA), one of such countries with a high trauma caseload. Through an international collaborative program [12], we have already used REBOA in several trauma cases in the RSA over the last years.

Our results provide evidence that a standard guidewire only adds minimal extra stiffness to the catheter, so this should not be an argument to choose an over-the-wire device. Moreover, guidewire-free devices itself are generally stiffer than the over-the-wire catheters and are provided with a flexible atraumatic tip to prevent aortic punctures. With the advent of REBOA-specific devices that house features of wire stiffness within the catheter, it is no longer advisable to use over-the-wire catheters for REBOA in emergency situations, especially those in which real-time fluoroscopy is not available. When fluoroscopy is available, an over-the-wire device can be considered to aid and confirm correct positioning, especially in middle-aged and elderly patients in whom tortuosity of blood vessels is more prevalent. REBOA via brachial artery access should not be performed without guidewire and fluoroscopic guidance given the higher risk of misplacement of the catheter. Of the over-the-wire devices, the Q50 ${ }^{\circledR}$ has several features that facilitate use and reduce the risk of malplacement or vessel damage (medium stiff, sufficient balloon diameter, flexible tip), especially when combined with the VacLok ${ }^{\circledR}$ syringe.

The tested catheters are small caliber catheters ranging from 6 to $10 \mathrm{Fr}$. Required introducer sheath size, however, varies from 6 to $22 \mathrm{Fr}$ with the LeMaitre ${ }^{\circledR} 45 \mathrm{~mm}$ Aortic Occlusion Catheter and Edwards Fogarty ${ }^{\circledR}$ 62080822F Occlusion Catheter, or even insertion via surgical cut-down only (Boston Scientific Equalizer ${ }^{\mathrm{TM}}$ with $40 \mathrm{~mm}$ balloon). The difference in catheter diameter and required sheath size is due to a larger diameter of the balloon or balloon wrapping, or to an increased balloon diameter after inflation and 
deflation of the devices. Devices compatible with a small introducer sheath are the ER-REBOA ${ }^{\mathrm{TM}}$ (7 Fr), Tokai Rescue Balloon $^{\mathrm{TM}}$ (7 Fr), and REBOA Medical AS REBOA Balloon ${ }^{\circledR} 15$ and 20 (6 Fr and $7 \mathrm{Fr}$, respectively). It is known that larger sheath sizes require either surgical repair of the access site after sheath removal or access site closure with a large-bore vascular closure device (VCD), while the smaller ones can be removed without surgical repair. Percutaneous access and access site closure might be elegant considering it reduces wound complications at the access site [13, 14]. Also, femoral access site complications, such as dissection, pseudo-aneurysm, embolism or limb ischemia are associated with the larger sheath sizes $[15,16]$; whereas, Teeter et al. [17] describe no access-related complications in their series with 7-Fr introducer sheaths. Hence, in the era of new, small diameter REBOA-specific devices, it is no longer advisable to use a catheter larger than $7 \mathrm{Fr}$ to perform this procedure in emergent situations, especially those in which vascular surgery consultation is not readily available. Furthermore, some large-bore VCDs such as the Perclose Proglide ${ }^{\circledR}$ and Prostar XL ${ }^{\circledR}$ (Abbott Vascular, Santa Clara, CA, USA) or the MANTA ${ }^{\mathrm{TM}}$ VCD (Teleflex Incorporated, Wayne, PA, USA) predominantly require either a preclose technique or pre-procedural depth measurement, thereby increasing time to vascular access, while early and quick vascular access and bleeding control is essential to improve outcome of the hemorrhagic trauma patient. The InClosure VCD (InSeal Medical, Caesarea, Israel) and PerQseal (Vivasure Medical, Galway Ireland) do not require any preparation before the main procedure. With the smaller sheath sizes, the femoral artery puncture site can be closed either with direct pressure or with an Angio-Seal ${ }^{\mathrm{TM}}$ (up to $8 \mathrm{Fr}$; Terumo, Tokyo, Japan) or EXOSEAL ${ }^{\circledR}$ or MYNXGRIP ${ }^{\circledR}$ (up to 7 Fr; Cordis, Santa Clara, CA, USA). These VCDs do not require any preprocedural preparations. Khan et al. described an alternative route for vascular access [18]. In their case, the aorta was punctured directly to place an ER-REBOA ${ }^{\mathrm{TM}}$ during laparotomy of a patient in extremis from multicavity penetrating trauma. With this hybrid approach quick vascular access is achieved without making unnecessary additional wounds. Their technique was successful in achieving hemodynamic stability.

Another feature that has to be taken into account is the balloon diameter. Maximum balloon diameter of the devices varies from $15 \mathrm{~mm}$ (REBOA Balloon $\left.{ }^{\circledR} 15\right)$ to $50 \mathrm{~mm}\left(\mathrm{Q} 50^{\circledR}\right.$ Stent Graft Balloon Catheter). The diameter of the occlusion balloon must be sufficient for total aortic occlusion, considering a smaller diameter is required for zone III occlusion than for zone I occlusion. Since most catheters carry a compliant balloon, the larger balloon diameters are also suitable for smaller aortic diameters. The REBOA Balloon ${ }^{\circledR}$ from REBOA Medical AS is the only device with a noncompliant balloon. Since non-compliant balloons have standard injection volumes, it may avoid over-inflation and subsequent rupture of the balloon or aortic wall. However, if the diameter of the aorta is smaller than the diameter of the non-compliant balloon, the balloon can create a tear in the aortic wall [19]. Furthermore, when the diameter of the aorta exceeds the diameter of the non-compliant balloon, no proper hemorrhage control will be established. The choice for a compliant or non-compliant balloon should be carefully considered based on the possible advantages and disadvantages and preference of the providing physician. Profound data on balloon material properties, such as compliance and the frictional properties of balloon surface, are missing and should be a topic of interest in the development of new, REBOA-specific devices.

All catheters have features that ease correct positioning. When fluoroscopy is available, radiopaque marker bands located at the balloon assist in positioning of the balloon. Especially, markers at the distal and proximal end of the balloon are helpful to visualize the proper inflation location. Catheters without markers located at the balloon but with a completely radiopaque catheter shaft are also helpful in positioning the balloon, assuming the balloon is near the distal end of the catheter. However, the exact occlusion zone cannot be confirmed unless balloon inflation occurs with a contrast medium. Consequently, inflation could occur in or overlapping the wrong aortic zone.

When fluoroscopy is not available, as in out-of-hospital REBOA or other resource-limited settings, devices with external length marks on the catheter shaft enable correct positioning of the balloon without imaging. Placement depths can be estimated before insertion of the catheter using anatomical landmarks such as the suprasternal notch, mid-sternum, xiphoid process or umbilicus [20, 21]. The ER-REBOA $^{\mathrm{TM}}$ facilitates accurate positioning with a length mark every $\mathrm{cm}$ and the exact length indicated with numbers every $5 \mathrm{~cm}$. The Tokai Rescue Balloon ${ }^{\mathrm{TM}}$, LeMaitre ${ }^{\circledR}$ Aortic Occlusion Catheters and Edwards Fogarty ${ }^{\circledR}$ Occlusions Catheters have length marks every 5 or $10 \mathrm{~cm}$ to assist positioning without fluoroscopy. The REBOA Balloon ${ }^{\circledR}$ has length marks at 30 and $55 \mathrm{~cm}$ to allow a standardized placement depth. It thereby might reduce procedure time. Standardized placement depth and the use of anatomical landmarks are based on average body measures from predominantly low-volume studies [20-24]. It may, however, lead to inadequate balloon positioning in an unknown number of patients. Therefore, future research should focus on further objectifying these population-based average body measures to help reduce the chance of improper balloon positioning in the field.

There are limitations in this study. The bending stiffness of the catheters is measured at room temperature and not at body temperature. Material properties may alter in higher temperatures; thus, bending stiffness might differ at body 
temperature. However, room temperature lies within the temperature range indicated on the catheters. In vivo longitudinal or axial stiffness can also be important; whereas in our study, bending stiffness was measured perpendicularly. However, the axial force from the catheter on the inflated balloon is in most cases negligible compared to the friction force between balloon and the inner wall of the artery, because of the large aspect ratio of the catheter. Also, a number of AOB are missing from this overview due to various reasons. To avoid a disparate comparison due to missing information, we chose to exclude these AOB. In addition, some of the devices might not be available in all countries due to regulatory restrictions.

\section{Conclusion}

Although there is a wide variety of $\mathrm{AOB}$ available, in resource-limited settings, a medium-stiff to stiff device that can be placed without guidewire and fluoroscopy guidance is warranted. Catheters such as the ER-REBOA ${ }^{\mathrm{TM}}$, Fogarty ${ }^{\circledR}$, and LeMaitre ${ }^{\circledR}$ are, therefore, advised. Of these devices, the ER-REBOA $^{\text {TM }}$ is the only catheter compatible with a small 7-Fr sheath and specifically designed for hemorrhage control in emergent settings and should, therefore, be preferred. Complete procedure kits for the REBOA Balloon ${ }^{\circledR}$ or ERREBOA $^{\mathrm{TM}}$ are beneficial, especially in environments with limited equipment resources. When fluoroscopy is available, an over-the-wire device should be considered to aid and confirm correct positioning. Of the over-the-wire devices, the Q50 ${ }^{\circledR}$ has several features that facilitate use and reduce the risk of malplacement or vessel damage.

Acknowledgements We acknowledge Cook Medical, Bloomington, IN, USA; Boston Scientific, Marlborough, MA, USA; Prytime Medical Devices, Boerne, TX, USA; Edwards Lifesciences, Irvine, CA, USA; LeMaitre Vascular, Burlington, MA, USA; Merit Medical, South Jordan, UT, USA; REBOA Medical AS, Norway; Medtronic, Minneapolis, MN, USA; Tokai Medical Products, Aichi, Japan; Minimally Invasive Technologies, Moscow, Russia, for providing the catheters used for this study. We would like to thank Jeroen Scheelings, Medical photographer, Alrijne Hospital, Leiderdorp, The Netherlands for assistance with photographs.

Author contributions SMV, BLSBB, PJEMV, GAHK, TER and RH prepared the study set-up. SMV and PJEMV developed the test bench and collected the data. SMV, BLSBB and RH prepared the manuscript. SMV and PJEMV prepared the tables and figures. SMV, BLSBB, PJEMV, GAHK, TER and RH contributed to the final version of the paper.

Funding This study was partly funded by the SZVK, the Dutch Ministry of Defense and the Karel Doorman Fund.
Data availability The data that support the findings of this study are available from the corresponding author, SMV, upon reasonable request.

\section{Compliance with ethical standards}

Conflict of interest Cook Medical, Bloomington, IN, USA; Boston Scientific, Marlborough, MA, USA; Prytime Medical Devices, Boerne, TX, USA; Edwards Lifesciences, Irvine, CA, USA; LeMaitre Vascular, Burlington, MA, USA; Merit Medical, South Jordan, UT, USA; REBOA Medical AS, Norway; Medtronic, Minneapolis, MN, USA; Tokai Medical Products, Aichi, Japan; Minimally Invasive Technologies, Moscow, Russia, provided the catheters used for this study. No other support was provided. S.M. Vrancken, B.L.S. Borger van der Burg, P.J.E.M. Vrancken, G.A. Kock and R. Hoencamp report no proprietary or commercial interest in any product mentioned or concept discussed in this article. Dr. Rasmussen is an inventor of REBOA and REBOA-like technology and is part of patents that have been declared and approved in several countries in this topic area. He has no relevant financial relationships to medical device industry in this topic area to disclose. The authors declare that there are no conflicts of interest that could inappropriately influence (bias) their work. We confirm that this submission has not been published elsewhere.

\section{References}

1. Brenner M, Bulger EM, Perina DG, Henry S, Kang CS, Rotondo $\mathrm{MF}$, et al. Joint statement from the American College of Surgeons Committee on Trauma (ACS COT) and the American College of Emergency Physicians (ACEP) regarding the clinical use of Resuscitative Endovascular Balloon Occlusion of the Aorta (REBOA). Trauma Surg Acute Care Open. 2018;3(1):e000154. https://doi.org/10.1136/tsaco-2017-000154.

2. Davidson AJ, Russo RM, Reva VA, Brenner ML, Moore LJ, Ball $\mathrm{C}$, et al. The pitfalls of resuscitative endovascular balloon occlusion of the aorta. J Trauma Acute Care Surg. 2018;84(1):192-202. https://doi.org/10.1097/ta.0000000000001711.

3. Stannard A, Eliason JL, Rasmussen TE. Resuscitative endovascular balloon occlusion of the aorta (REBOA) as an adjunct for hemorrhagic shock. J Trauma. 2011;71(6):1869-72. https://doi. org/10.1097/TA.0b013e31823fe90c.

4. Dunn EL, Moore EE, Moore JB. Hemodynamic effectsof aortic occlusion during hemorrhagic shock. Ann Emerg Med. 1982;11(5):238-41. https://doi.org/10.1016/s0196-0644(82)80090 -5 .

5. Markov NP, Percival TJ, Morrison JJ, Ross JD, Scott DJ, Spencer JR, et al. Physiologic tolerance of descending thoracic aortic balloon occlusion in a swine model of hemorrhagic shock. Surgery. 2013;153(6):848-56. https://doi.org/10.1016/j.surg.2012.12.001.

6. White JM, Cannon JW, Stannard A, Markov NP, Spencer JR, Rasmussen TE. Endovascular balloon occlusion of the aorta is superior to resuscitative thoracotomy with aortic clamping in a porcine model of hemorrhagic shock. Surgery. 2011;150(3):400-9. https ://doi.org/10.1016/j.surg.2011.06.010.

7. Rasmussen TE, Franklin CJ, Eliason JL. Resuscitative Endovascular Balloon Occlusion of the Aorta for hemorrhagic shock. JAMA Surgery. 2017;152(11):1072-3. https://doi.org/10.1001/ jamasurg.2017.3428.

8. Ribeiro Junior MAF, Feng CYD, Nguyen ATM, Rodrigues VC, Bechara GEK, De-Moura RR, et al. The complications associated with Resuscitative Endovascular Balloon Occlusion of the Aorta 
(REBOA). World J Emerg Surg. 2018. https://doi.org/10.1186/ s13017-018-0181-6.

9. Onishi Y, Kimura H, Kanagaki M, Oka S, Fukumoto G, Otani $\mathrm{T}$, et al. Loop formation by an aortic occlusion balloon catheter during resuscitative endovascular balloon occlusion of the aorta (REBOA). Radiol Case Rep. 2019;14(2):184-6. https://doi. org/10.1016/j.radcr.2018.10.018.

10. Borger van der Burg BLS, Van Schaik J, Brouwers JJWM, Wong CY, Rasmussen TE, Hamming JF, et al. Migration of aortic occlusion balloons in an in vitro model of the human circulation. Injury. 2019;50(2):286-91. https://doi.org/10.1016/j.injury.2018.12.026.

11. Hörer TM, Cajander P, Jans A, Nilsson KF. A case of partial aortic balloon occlusion in an unstable multi-trauma patient. Trauma. 2016;18(2):150-4. https://doi.org/10.1177/1460408615624727.

12. van der Wal H, van Dongen TTCF, Vermeulen CFW, Bruce JL, Bekker W, Manchev V, et al. Developing a blueprint for a civilian-military collaborative program in trauma training for northern European countries: a South African experience. Injury. 2020;51(1):70-5. https://doi.org/10.1016/j.injury.2019.07.034.

13. Buck DB, Karthaus EG, Soden PA, Ultee KH, van Herwaarden JA, Moll FL, et al. Percutaneous versus femoral cutdown access for endovascular aneurysm repair. J Vasc Surg. 2015;62(1):16211. https://doi.org/10.1016/j.jvs.2015.01.058.

14. Nakamura M, Chakravarty T, Jilaihawi H, Doctor N, Dohad S, Fontana G, et al. Complete percutaneous approach for arterial access in transfemoral transcatheter aortic valve replacement: a comparison with surgical cut-down and closure. Catheter Cardiovasc Interv. 2014;84(2):293-300. https://doi.org/10.1002/ ccd.25130.

15. DuBose JJ, Scalea TM, Brenner M, Skiada D, Inaba K, Cannon J, et al. The AAST prospective Aortic Occlusion for Resuscitation in Trauma and Acute Care Surgery (AORTA) registry: data on contemporary utilization and outcomes of aortic occlusion and resuscitative balloon occlusion of the aorta (REBOA). J Trauma Acute Care Surg. 2016;81(3):409-19. https://doi.org/10.1097/ TA.0000000000001079.

16. Morrison JJ, Galgon RE, Jansen JO, Cannon JW, Rasmussen TE, Eliason JL. A systematic review of the use of resuscitative endovascular balloon occlusion of the aorta in the management of hemorrhagic shock. J Trauma Acute Care Surg. 2016;80(2):324-34. https://doi.org/10.1097/TA.0000000000000913.
17. Teeter WA, Matsumoto J, Idoguchi K, Kon Y, Orita T, Funabiki $\mathrm{T}$, et al. Smaller introducer sheaths for REBOA may be associated with fewer complications. J Trauma Acute Care Surg. 2016;81(6):1039-45. https://doi.org/10.1097/TA.0000000000 001143.

18. Khan MZ, Bruce J, Baer D, Hoencamp R. Hybrid use of REBOA in a South African tertiary trauma unit for penetrating torso trauma. BMJ Case Rep. 2019. https://doi.org/10.1136/bcr-2019229538.

19. Sovik E, Stokkeland P, Storm BS, Asheim P, Bolas O. The use of aortic occlusion balloon catheter without fluoroscopy for life-threatening post-partum haemorrhage. Acta Anaesthesiol Scand. 2012;56(3):388-93. https://doi.org/10.111 $1 /$ j.1399-6576.2011.02611.x.

20. Linnebur M, Inaba K, Haltmeier T, Rasmussen TE, Smith J, Mendelsberg R, et al. Emergent non-image-guided resuscitative endovascular balloon occlusion of the aorta (REBOA) catheter placement: a cadaver-based study. J Trauma Acute Care Surg. 2016;81(3):453-7. https://doi.org/10.1097/TA.000000000000110 6.

21. Okada Y, Narumiya H, Ishi W, Iiduka R. Anatomical landmarks for safely implementing resuscitative balloon occlusion of the aorta (REBOA) in zone 1 without fluoroscopy. Scand J Trauma Resusc Emerg Med. 2017;25(1):63. https://doi.org/10.1186/s1304 9-017-0411-z.

22. MacTaggart JN, Poulson WE, Akhter M, Seas A, Thorson K, Phillips NY, et al. Morphometric roadmaps to improve accurate device delivery for fluoroscopy-free resuscitative endovascular balloon occlusion of the aorta. J Trauma Acute Care Surg. 2016;80(6):941-6. https://doi.org/10.1097/TA.000000000000104 3.

23. Morrison JJ, Stannard A, Midwinter MJ, Sharon DJ, Eliason JL, Rasmussen TE. Prospective evaluation of the correlation between torso height and aortic anatomy in respect of a fluoroscopy free aortic balloon occlusion system. Surgery. 2014;155(6):1044-51. https://doi.org/10.1016/j.surg.2013.12.036.

24. Pezy P, Flaris AN, Prat NJ, Cotton F, Lundberg PW, Caillot JL, et al. Fixed-distance model for balloon placement during fluoroscopy-free resuscitative endovascular balloon occlusion of the aorta in a civilian population. JAMA Surg. 2017;152(4):351-8. https://doi.org/10.1001/jamasurg.2016.4757.

\section{Affiliations}

\section{Suzanne M. Vrancken ${ }^{1}$ (D) - Boudewijn L. S. Borger van der Burg ${ }^{1}$ - Paul J. E. M. Vrancken ${ }^{1}$. Gert-Aldert H. Kock ${ }^{2}$. Todd E. Rasmussen ${ }^{3}$ Rigo Hoencamp ${ }^{1,4,5,6}$}

1 Department of Surgery, Alrijne Hospital Leiderdorp, Simon Smitweg 1, 2353GA Leiderdorp, The Netherlands

2 Department of Radiology, Alrijne Hospital, Leiderdorp, The Netherlands

3 The Department of Surgery, The Uniformed Services University and Walter Reed National Military Medical Center, Bethesda, MD, USA
4 Defense Healthcare Organization, Ministry of Defense, Utrecht, The Netherlands

5 Leiden University Medical Centre, Leiden, The Netherlands

6 Trauma Research Unit, Department of Surgery, Erasmus MC, University Medical Center Rotterdam, Rotterdam, The Netherlands 